\title{
El juego temático de roles sociales: aportes al desarrollo en la edad preescolar
}

\author{
0 jogo temático de papeis sociais na educação infantil \\ The Thematic Set of Social Roles: Contributions to Development in Preschool
}

\author{
Claudia Ximena González Moreno* \\ Pontificia Universidad Javeriana, Universidad Iberoamericana de Puebla \\ Yulia Solovieva, Luis Quintanar Rojas \\ Benemérita Universidad Autónoma de Puebla
}

Doi: dx.doi.org/10.12804/ap132.2.2014.08

\section{Resumen}

El juego temático de roles sociales es importante en la educación inicial porque posibilita el desarrollo de la actividad simbólica, voluntaria, reflexiva, comunicativa desplegada e imaginativa. Estas son formaciones psicológicas que garantizan el aprendizaje en la edad escolar. En este artículo se presenta una reflexión acerca de la necesidad de utilizar el juego temático de roles sociales en las aulas de preescolar, ${ }^{1}$ por su efecto en la promoción de habilidades y en la prevención de dificultades del desarrollo y del aprendizaje. Además, esta actividad se considera relevante porque posibilita que los niños adquieran las herramientas necesarias para la vida y para actuar de manera activa en la sociedad. Asimismo, contribuye a lograr el bienestar de los preescolares y, por consiguiente, a mejorar el derecho a la educación de calidad en el marco del desarrollo humano y de la salud escolar. ${ }^{2}$

Palabras clave: Juego temático de roles sociales, desarrollo psicológico infantil, zona de desarrollo próximo, interiorización, prevención de dificultades del aprendizaje, calidad de la educación

1 En Colombia, las acepciones educación inicial, educación para la primera infancia y educación preescolar se usan como sinónimos. Según lo establecido en la Ley 115 de 1994, y en cumplimiento de lo dispuesto en los artículos 67 y 68 de la Constitución Nacional, en Colombia la educación es un derecho y un servicio público, y puede ser ofrecida por el sector oficial o por particulares. Las disposiciones actuales sobre el sistema educativo en el país están amparadas también por la Ley 1098 de 2006, mejor conocida como la Ley de la Infancia y la Adolescencia, en la que se reconoce a niños y adolescentes como sujetos titulares de derechos. Estos derechos fueron establecidos en la Convención Internacional de los Derechos del Niño. Específicamente en el artículo 29 se estipula como derecho de la Primera Infancia la educación inicial. El nivel de preescolar comprende los grados de pre-jardín, jardín y transición, y atiende a niños desde los tres a los cinco años, de acuerdo con la reglamentación del Decreto 2247 de 1997. Ver Consejo Nacional de Política Económica Social, República de Colombia, Departamento Nacional de Planeación, Política Pública Nacional de Primera Infancia. Colombia por la primera infancia. Documento Conpes Social. Ministerio de la Protección Social, Ministerio de Educación Nacional, Instituto Colombiano de Bienestar Familiar, Bogotá. Recuperado de http:// cms-static.colombiaaprende.edu.co/cache/binaries/articles-177828_archivo_pdf_conpes109.pdf?binary_rand=3435

2 La salud escolar articula las necesidades de los niños en el ámbito de la salud y la educación con las habilidades para la vida en pro del desarrollo humano. Ver Colombia aprende. La red del conocimiento. Preescolar, básica y media. (2011). Sistema educativo en Colombia. Bogotá: Ministerio de Educación Nacional. Recuperado de http://www.colombiaaprende.edu.co/html/home/1592/article-235863.html

* Claudia Ximena González Moreno, Facultad de Educación, Pontificia Universidad Javeriana, Universidad Iberoamericana de Puebla; Yulia Solovieva, Maestría en Diagnóstico y Rehabilitación Neuropsicológica, Benemérita Universidad Autónoma de Puebla; Luis Quintanar Rojas, Maestría en Diagnóstico y Rehabilitación Neuropsicológica, Benemérita Universidad Autónoma de Puebla.

La correspondencia relacionada con este artículo debe ser dirigida a Claudia Ximena González Moreno, Facultad de Educación, Pontificia Universidad Javeriana, Carrera 7 \# 40-62, edificio 25. Correo electrónico: c.gonzalezm@javeriana.edu.co

Para citar este artículo: González, M. C., Solovieva, Y., Quintanar, R. L. (2014). El juego temático de roles sociales: aportes al desarrollo en la edad preescolar. Avances en Psicología Latinoamericana, 32(2), 287-308. doi: dx.doi.org/10.12804/apl32.2.2014.08 


\section{Resumo}

O jogo temático de papeis sociais é importante na educação inicial porque possibilita o desenvolvimento das da atividade simbólica, voluntária, reflexiva, comunicativa, aberta e imaginativa. Estas são formações psicológicas que garantem a aprendizagem na idade escolar. Neste artigo apresenta-se uma reflexão acerca da necessidade de utilizar o jogo temático de papeis sociais nas salas de aula da educação infantil ${ }^{3}$ por seu efeito na promoção de habilidades e na prevenção de dificuldades do desenvolvimento e da aprendizagem. Além disso, esta atividade considera-se relevante porque possibilita que as crianças adquiram ferramentas necessárias para a vida e para atuar de forma ativa na sociedade. Igualmente, contribui a conseguir o bem-estar das crianças $\mathrm{e}$, por conseguinte, a melhorar o direito à educação de qualidade no marco do desenvolvimento humano e da saúde escolar. ${ }^{4}$

Palavras-chave: Jogo temático de papeis sociais, desenvolvimento psicológico infantil, zona de desenvolvimento próximo, interiorização, prevenção de dificuldades da aprendizagem, qualidade da educação

\section{fibstract}

The thematic set of social roles is important in early childhood education because it enables the development of symbolic, voluntary, reflective, communicative and openly imaginative activities. These are forms of psychological education to ensure success of learning at school age. This paper presents a reflection on the need for thematic role play in preschool classrooms ${ }^{5}$ because of its effect furthering skills and preventing difficulties in development and learning. Furthermore, this activity is considered important because it enables children to acquire tools necessary for life and to act actively in society. It also contributes to the well being of preschoolers and thus to improve the right to quality education in the context of human development and school health. ${ }^{6}$

Keywords: Game themed social roles, child psychological development, ZPD, internalization, quality of education.

Varios especialistas en psicología, neuropsicología y pedagogía coinciden en reconocer la importancia que tiene el juego para el desarrollo infantil (Bonilla, Solovieva \& Jiménez, 2012; Bodrova, 1998; Bodrova \& Leong, 1996, 2001, 2003, 2010; Bredikyte, 2004; Bredikyte \& Hakkarainen, 2007; Elkonin, 1980; Solovieva \& Quintanar, 2012; Vigotsky, 1984). La investigación sobre el desarrollo muestra que cuando los niños juegan adecuadamente en el preescolar, el juego contribuye al aprendizaje en la edad escolar (Bergen, 2002).

3 Na Colômbia, as acepções educação inicial, educação para a primeira infância e educação pré-escolar usam-se como sinônimos. Segundo o estabelecido na Lei 115 de 1994, e em cumprimento do disposto nos artigos 67 e 68 da Constituição Nacional, na Colômbia a educação é um direito e um serviço público, e pode ser oferecida pelo setor oficial ou por particulares. As disposições atuais sobre o sistema educativo no país estão amparadas também pela Lei 1098 de 2006, melhor conhecida como a Lei da Infância e a Adolescência, na que se reconhece a crianças e a adolescentes somo sujeitos titulares de direitos. Estes direitos foram estabelecidos na Convenção Internacional dos Direitos da Criança. Especificamente no artigo 29 estipula-se como direito da Primeira Infância a educação inicial. O nível de Educação Pré-escolar compreende os graus de creche, pré-escola e jardim de infância, e atende a crianças desde os três aos cinco anos, de acordo com a regulamentação do Decreto 2247 de 1997. Ver Conselho Nacional de Política Econômica Social, República da Colômbia, Departamento Nacional de Planejamento, Política Pública Nacional de Primeira Infância. Colômbia pela Primeira Infância. Documento Conpes Social. Ministério da Proteção Social, Ministério de Educação Nacional, Instituto Colombiano de Bem-estar Familiar, Bogotá. Recuperado de http://cms-static. colombiaaprende.edu.co/cache/binaries/articles-177828 archivo pdf conpes109.pdf?binary rand=3435

4 A saúde escolar articula as necessidades das crianças no âmbito da saúde e a educação com as habilidades para a vida em pro do desenvolvimento humano. Ver "Colômbia aprende". A rede de conhecimento. Pré-escolar, ensino básico e ensino médio. (2011). Sistema educativo na Colômbia. Bogotá: Ministério de Educação Nacional. Recuperado de http://www.colombiaaprende.edu.co/html/home/1592/article-235863.html

5 The right to quality education is undoubtedly one of the most important rights of children. As set out in Law 115 of 1994 , and pursuant to the provisions of Articles 67 and 68 of the Constitution, in Colombia education is a right and a public service, and may be provided by the public sector or by private. The current provisions on the education system in the country are also covered by Law 1098 of 2006 , better known as the Law of Children and Adolescents, which recognizes children and adolescents as rights holders. These rights were established in the Convention on the Rights of the Child. Specifically Article 29 stipulates as law Early Childhood early education. The preschool level comprises grades pre-kindergarten, garden and transition, and serves children from three to five years, according to the regulations of Decree 2247 of 1997. http://www.colombiaaprende.edu.co/html/home/1592/article-235863.html

6 School health articulates the needs of children in the field of health and education with life skills in human development. 
Un indicador del desarrollo psicológico del niño y de su preparación para la escuela tiene que ver con cómo juega (Solovieva \& Quintanar, 2012). Por esta razón, Elkonin (1980) afirma que algunas preguntas clave en educación infantil son ¿qué tanto ha jugado el niño? y ¿cómo lo hace?

En Colombia, en el Lineamiento Pedagógico y Curricular para la Educación Inicial en el Distrito (2010) se reconoce el valor del juego para el progreso infantil en la edad preescolar. Esta edad es de gran importancia para el desarrollo posterior del niño en la etapa escolar. No obstante, en la práctica dentro del aula de clase se da más valor a la introducción de la lectura y la escritura sin reflexionar en el desarrollo del niño a nivel psicológico.

Los educadores han considerado siempre el juego como un elemento básico en la primera infancia. Sin embargo, la creciente demanda de resultados mensurables para preescolares está llevando el juego a la periferia del currículo, dedicando más tiempo al contenido académico. Los maestros admiten que los beneficios del juego no son tan fáciles de entender y evaluar como, por ejemplo, la capacidad de los niños de reconocer las letras y escribir sus nombres (Bodrova \& Leong, 2003, p. 1).

En los resultados del último estudio Internacional de Competencia Lectora (PIRLS), ${ }^{7}$ en el 2011, se encontró que seis de cada diez estudiantes de primaria en Colombia tienen dificultades para entender textos. El país participó con 4000 niños de cuarto grado de 150 colegios públicos y pri- vados. Esto trae como consecuencia dificultades para orientar el proyecto de desarrollo personal y participar en la sociedad; así como dificultades del aprendizaje, bajo rendimiento académico y frustración escolar que muchas veces lleva a la deserción.

En Colombia, se pretende lograr que la educación dé respuesta satisfactoria a demandas sociales que son inaplazables. Se trata de ofrecer una educación de calidad ${ }^{8}$ desde la etapa preescolar.

La calidad de la educación está relacionada con la relevancia. En la medida que se crece en relevancia se crece en calidad. Además la educación de calidad es un factor de transformación social. La calidad de educación claramente se entiende como la capacidad de proporcionar a los estudiantes el dominio de los códigos culturales básicos, las capacidades para la participación democrática y ciudadana, el desarrollo de la capacidad para resolver problemas y seguir aprendiendo, y el desarrollo de valores y actitudes acordes con una sociedad que desea una vida de calidad para todos sus habitantes (Schmelkes, 1995, p. 4).

El índice de bajo desempeño escolar está asociado con el bajo desarrollo de las actividades simbólica, voluntaria, reflexiva, comunicativa desplegada e imaginativa (Akhutina, 2002; Akhutina \& Pilayeva, 2012; Bonilla, 2009; Davidov, 1996; Hetzer, 1926; Lázaro, 2009; Quintanar \& Solovieva, 2007; Solovieva \& Quintanar, 2007 y Solovieva \& Quintanar, 2010). En este artículo, se reconoce que esta situación podría cambiar si se empieza a utilizar de forma adecuada el juego temático de

7 PIRLS (Progress in International Reading Literacy Study), es un estudio de la Asociación Internacional para la Evaluación del Rendimiento Educativo (IEA). Fundada en 1959, la IEA ha dirigido, en los últimos 45 años, estudios sobre las políticas, las prácticas y los resultados educativos en más de sesenta países de todo el mundo.

8 "La calidad de la educación está constituida por al menos cuatro componentes. El primero de ellos es precisamente la relevancia. No puede hablarse de calidad educativa si el sistema educativo (o el nivel, o la zona, o la escuela) no está siendo capaz de propiciar el desarrollo de aprendizajes relevantes, útiles, relacionados con la vida actual y futura de los alumnos. El segundo es el concepto de eficacia, que se refiere a la capacidad de un sistema educativo de lograr los objetivos que explícitamente se propone suponiendo, claro está, que estos sean relevantes, con todos sus alumnos. El tercero es la equidad, lo que supone el reconocimiento de la diversidad en todos sus sentidos -de características individuales de los alumnos, del lugar donde viven, de las condiciones socioeconómicas de sus familias, de las características culturales de sus padres, de la etnia a la que pertenecen, de la comunidad en la que viven-. El componente de equidad supone el reconocimiento de que todos estos factores inciden sobre las oportunidades de escolaridad y aprendizaje. Por tanto, la equidad significa que, a fin de ser eficaces - de obtener los objetivos propuestos con todos los alumnos- es necesario apoyar de manera diferencial a los alumnos, escuelas, zonas, que más lo necesiten. Supone, en otras palabras, dar más a los que menos tienen. Por último, el cuarto componente, de naturaleza diferente a todos los anteriores es el de eficiencia. Este componente se refiere a lograr calidad en la medida que se logran resultados similares con los mismos recursos" (Schmelkes, 1997, p. 10). 
roles sociales en el preescolar, así como lo propone la psicología pedagógica histórico-cultural (Akhutina \& Pilayeva, 2012; Elkonin, 1980; Solovieva \& Quintanar, 2012).

\begin{abstract}
No obstante, con frecuencia se evalúan ciertas habilidades, cuya presencia no garantizan el éxito escolar. Esas mismas habilidades son las que se trabajan de manera forzada en el jardín de niños: la lectura, la escritura y las matemáticas. Lo anterior permite suponer que no se evalúa la presencia del verdadero indicador que señale el nivel de preparación del niño para la escuela. Al mismo tiempo, es posible que las actividades que se ejercen con gran insistencia en los jardines no sean las que garantizan el verdadero desarrollo del niño. De acuerdo con la teoría del desarrollo psicológico por etapas, existen actividades que son útiles y necesarias para cada edad infantil en particular. De la misma manera, no todas las actividades son útiles para todas las edades (Solovieva \& Quintanar, 2012, p. 10).
\end{abstract}

Se ha encontrado que muchos niños ni siquiera alcanzan el desarrollo complejo de juego temático de roles sociales en la edad preescolar (GonzálezMoreno, Solovieva \& Quintanar, 2009, 2011). Además, en estudios experimentales realizados por Salmina $(1988,1989,2001,2010)$ se identificó que "cuanto más bajo sea el nivel general de desarrollo del niño, menos desarrollo manifiesta su actividad lúdica". Es así como se considera que con frecuencia los preescolares no están preparados para enfrentar las nuevas habilidades en la edad escolar.

Según algunos investigadores, un número creciente de los niños no desarrolla las formas maduras de juego en el jardín infantil (Bodrova \& Leong, 2010; Bredikyte, 2004; Bredikyte \& Hakkarainen, 2007; Mikhailenko \& Korotkova, 2001). Una solución posible que se puede plantear es la intervención de los adultos de manera eficaz con el fin de apoyar el desarrollo de formas más maduras del juego de roles. Los criterios que indican que el juego ha madurado son que está basado en la imaginación, no es estereotipado, dura en el tiempo, de- manda acciones del más alto nivel, tiene estructura narrativa, los adultos y los niños asumen diferentes papeles, se involucra a los niños en el proceso de exploración activa experimentando y reflexionando (Bredikyte, 2004; Lindqvist, 1995). Los aspectos más significativos de una intervención exitosa son: (a) la motivación por un tema común; (b) la participación activa en el rol; (c) se evidencia el carácter dialógico en las interacciones.

Bredikyte y Hakkarainen (2007) en sus estudios encontraron que de temáticas reales, los niños pasan a desarrollar temas fantásticos. El juego se construye de manera colectiva entre las ideas de los niños y el adulto. En el juego, los niños aprenden a someter sus propios deseos a las exigencias que se plantean. El juego es una actividad colectiva que impulsa a seguir una serie de normas sociales. Asimismo, los niños siguen las reglas, se hace lo que el juego exige. La actividad lúdica garantiza un sistema de actividad que ayuda al niño a ampliar sus propios límites y posibilidades y contener otros, así se desarrolla la personalidad. En este proceso, el adulto requiere aprender el lenguaje del juego y tener una intensión verdadera para jugar. La actividad de juego es un medio que mueve a los participantes hacia la construcción de un sistema conjunto de la actividad y prepara el autodesarrollo.

En estudios con preescolares se ha identificado el estado deficiente de su desarrollo al ingresar a la escuela primaria, lo que motiva la necesidad de desplegar programas preventivos que garanticen el desarrollo de habilidades mínimas necesarias para hacer frente a la actividad escolar (Quintanar \& Solovieva, 2007; Talizina, 2000, 2009). Las habilidades que se consideran importantes en el preescolar son la actividad simbólica, la actividad voluntaria, la actividad reflexiva, la actividad comunicativa desplegada y la actividad imaginativa. Uno de los programas que tiene relevancia en el preescolar y que podría contribuir de forma importante a desarrollar estas habilidades es el juego temático de roles sociales. De esta manera, se puede ayudar a mejorar la calidad de vida ${ }^{9}$ de los niños.

9 "La calidad de vida es aspiración legítima de todo ser humano. Esta depende primordialmente de la calidad del quehacer humano y, en último término, de la calidad de los seres humanos. La riqueza de una nación depende de su gente. La función de la educación es crear seres humanos 
En estudios realizados por Lázaro y cols. (2009) se identificó el efecto de la aplicación de un programa de juego en el desarrollo de niños preescolares. En el estudio participaron treinta niños en cuatro grupos: el primer grupo integrado por niños de segundo grado regular, el segundo grupo con niños de segundo grado con necesidades especiales, el tercer grupo con niños de tercer grado regular y el cuarto grupo por niños de tercer grado con necesidades especiales. Los grupos con necesidades especiales presentaban pobre desarrollo verbal y problemas de regulación de su comportamiento. El programa duró 75 sesiones de 60 minutos. Se hicieron sesiones de intervención de acuerdo con las necesidades de los niños, considerando el juego objetal, simbólico desplegado y el juego temático de roles sociales. Durante la aplicación del programa se evidenciaron cambios graduales en todos los grupos que incluían las siguientes características: incremento en la atención de los niños, mejoría en la retención y comprensión de información, mayor organización, desenvolvimiento y seguridad durante las actividades, mayor respeto a las reglas y mayor motivación (Solovieva \& Quintanar, 2012). Esto indica que el programa de intervención que incluye el juego se dirige a las necesidades de los niños y tiene efectos positivos en el desarrollo infantil.

En otro estudio (González-Moreno, Solovieva \& Quintanar, 2009) se identificó el impacto de la actividad de juego temático de roles en la formación de la actividad reflexiva en niños de último nivel de preescolar en Colombia (48 niños entre los 5 y 6 años de una institución educativa regular privada). El estudio fue de diseño mixto, que incorpora elementos de diseño cuasi-experimental pre/post y tipo descriptivo. Los resultados mostraron que la inclusión de actividades por medio del programa de juego resultó ser efectiva para el desarrollo de las formaciones básicas de esta edad: conducta voluntaria y pensamiento reflexivo. Estos elementos en conjunto se constituyen en requisitos para la preparación de los niños para la escuela, así como para la adquisición de actividades complejas en la edad escolar (Quintanar \& Solovieva, 2005).

En esta investigación se evidenció: (a) cambio en la actitud hacia el aprendizaje, (b) aumento en la participación en la clase, (c) interés y motivación amplia frente a la actividad escolar, (d) efecto emocional positivo, (e) vínculos sociales entre los niños, (f) desarrollo de la habilidad para trabajar conjuntamente en busca de un objetivo, (g) incremento de la riqueza léxica, (h) el lenguaje pasó de cumplir una importante función en las relaciones sociales a transformarse en un instrumento de la organización psíquica interna del niño.

En estas investigaciones se consideran importantes las acciones pedagógicas desde las primeras etapas de escolaridad. De esta forma, se ayuda a que los niños alcancen un nivel de habilidades efectivas en la relación que establecen con ellos mismos, con los demás y con el mundo, principalmente con la familia, amigos y adultos significativos; se mejora su calidad de vida y se evitan problemas de aprendizaje y comunicación; también se impacta positivamente el desarrollo de su personalidad y se contribuye a que puedan enfrentar y afrontar con mayor facilidad los retos que impone el contexto socio-cultural.

En un estudio realizado por Bonilla (2013), se identificó el efecto de la aplicación de un programa de juego de roles en la formación de la función simbólica, a partir de las características identificadas antes y después de la aplicación del programa, en un grupo de niños de tercer grado de preescolar de

de calidad. La calidad de las personas es la primera preocupación de la filosofía de la calidad. Un sistema en el que se persigue la calidad se preocupará porque las personas desarrollen al máximo sus potencialidades. Las personas se desarrollan como tales cuando son capaces de crecer integralmente. Es necesario, para desarrollarse, tener conocimientos, gozar de una calidad de vida digna, ser respetados y aceptados. Pero quizás más importante que todo lo anterior, el proceso de desarrollo personal se encuentra en el descubrimiento del sentido de la vida que procede fundamentalmente de demostrarse a sí mismo la capacidad de transformar la realidad en el sentido en que uno cree que debe ser transformada y, de manera igualmente importante, en hacerlo en forma congruente con los valores que uno quiere ver reflejados en esa realidad que contribuye a transformar. El mejoramiento efectivo genera una verdadera satisfacción en la vida. La búsqueda de la calidad genera el espacio para que esto sea posible. Le da un sentido de transformación al trabajo cotidiano y persigue hacerlo resaltando el compromiso, la responsabilidad y la solidaridad con los seres humanos con los que se trabaja y con el objetivo colectivo en el que se participa. La filosofía de la calidad se basa en la convicción del deseo inherente que las personas tienen de lograr calidad y valor, de compartir su experiencia y de apoyarse uno al otro" (Schmelkes, 1994, p. 54). 
procedencia suburbana en Tlaxcala, México. Se trabajó con 57 niños (26 del grupo experimental y 31 del grupo control). Los resultados dejan ver que el programa de juego influyó en forma positiva en el desarrollo de la función simbólica, en las etapas de: sustitución, codificación y esquematización, en los niños del grupo experimental, presentando mejores ejecuciones en las tareas de evaluación, principalmente en los planos materializado, perceptivo concreto y esquematizado, que en el plano verbal, en comparación con los niños del grupo control.

Por lo anterior, el objetivo de este artículo es mostrar la importancia que tiene el juego temático de roles sociales como actividad característica de la edad preescolar al desarrollar las formaciones psicológicas: simbólica, voluntaria, reflexiva, comunicativa desplegada e imaginativa, que garantizan la organización óptima de la vida del niño en esta etapa del desarrollo.

\section{El juego temático de roles sociales en la transición de la edad preescolar a la escolar}

\footnotetext{
La frontera de las edades preescolar y escolar es de suma importancia para la actividad cognitiva del niño en las etapas posteriores de su desarrollo. De acuerdo con los datos psicológicos, neuropsicológicos y neurofisiológicos, dicha edad se considera como crítica. En esta edad, la consolidación de los mecanismos cerebrales de regulación de toda la actividad del niño tiene un significado particular. Precisamente en esta edad dichos mecanismos cerebrales alcanzan un cierto nivel de madurez, lo cual garantiza la selectividad y el aspecto voluntario de las funciones psicológicas superiores. Asimismo, se afirma que la actividad cerebral se conforma y desarrolla a partir de la actividad psicológica (Solovieva, Machinskaya, Quintanar, Bonilla \& Pelayo, 2009, p. 7).
}

La edad preescolar es diferente a la escolar, como lo plantea Talizina (2009). En la edad preescolar el niño adquiere conceptos cotidianos (empíricos), de manera inmediata por medio del contacto físico, durante su participación en diversas actividades como los juegos, los paseos, la actividad práctica y artística. En la edad escolar, el niño adquiere conceptos científicos (teóricos). Estos no se obtienen por medio de la simple interacción con los objetos, no se construyen espontáneamente sobre la base de la experiencia cotidiana del niño. Para la formación de los conceptos científicos, el maestro debe presentar claramente los componentes (características) esenciales del concepto con el que trabaja. Estas propiedades resultan de los procesos de abstracción y generalización de las características esenciales de los objetos (Talizina, 2000, 2009).

Para que el niño pueda pasar con éxito de la etapa preescolar a la escolar necesita participar; de forma desplegada y profunda, en el juego temático de roles sociales (Bredikyte \& Hakkarainen, 2007, Solovieva \& Quintanar, 2012). De esta manera, se evita el fracaso y las dificultades del aprendizaje.

En el enfoque histórico-cultural y la teoría de la actividad, el juego temático de roles es una forma particular de adquisición de la experiencia social y cultural (Vigotsky, 1984). Por esto se dice que el juego de roles tiene un origen social (Elkonin, 1980). Al principio, la actividad lúdica se presenta a partir de la acumulación de las intenciones del adulto, quien propone el juego al niño (Solovieva \& Quintanar, 2012). Aquí, el juego es atractivo por medio de la actividad conjunta que se comparte. El niño interactúa con objetos, considerando propósitos específicos e intencionales y después usa sustitutos de objetos. Luego, él empieza a influir en su propia conducta y en la de los demás a partir de su propio lenguaje verbal externo. La motivación que surge en este proceso es importante para el desarrollo de la personalidad porque le permite al niño aprender a comportarse de acuerdo con la situación. Así surge la orientación hacia las normas sociales.

En el juego temático de roles sociales nace el sentido de las acciones humanas a partir de las relaciones con otra persona. En esta actividad lúdica, el niño por primera vez se somete de manera placentera a las reglas del juego. Por ello es que el juego de roles constituye una actividad básica y necesaria para la formación de la conducta voluntaria y organizada, actividad en la cual el niño puede dirigir su atención. Así, el niño aprende a coordinar sus movimientos y se habitúa a dirigir sus actividades en correspondencia con las reglas 


\section{El juego temático de roles sociales: aportes al desarrollo en la edad preescolar}

conocidas (Bonilla, Solovieva, Figueroa, Martínez \&

Quintanar, 2004, p. 118).

El juego temático de roles sociales no surge espontáneamente, sino que requiere del apoyo del adulto en un primer momento para que así se puedan desarrollar en su forma compleja las neoformaciones,${ }^{10}$ características de la edad preescolar: simbólica, voluntaria, reflexiva, comunicativa desplegada e imaginativa. El juego libre es una situación que no aporta al desarrollo del niño (Lázaro, 2009; Solovieva \& Quintanar, 2012). Como se evidencia en la tabla 1, el juego libre no garantiza el nivel óptimo de aprendizaje en la escuela, especialmente en la lectura, la escritura y las matemáticas. Según estudios neuropsicológicos y psicológicos, el éxito en los proceso de lectura, escritura y matemáticas dependen de un nivel de desarrollo adecuado de las actividades simbólica, voluntaria, reflexiva, comunicativa desplegada y la imaginativa por medio del juego de roles (Elkonin, 1989, 1995; Obukhova, 1995, 2006; Salmina \& Filimonova, 2001; Salmina, 2010; Solovieva \& Quintanar, 2012; Vigotsky, 1995b, 1996a y b).

En la teoría de la actividad, el desarrollo se da solamente a partir de la participación conjunta del niño con el adulto y con otros niños en diferentes tipos de actividades. En educación inicial, el mayor significado lo tiene la actividad rectora porque promueve la aparición de las neoformaciones, las cuales son objetivos fundamentales de esta edad psicológica (Solovieva \& Quintanar, 2012). Cada edad tiene sus propios objetivos particulares. En este caso, en la educación inicial se presenta por medio del juego temático de roles sociales (Elkonin, 1980; Leontiev, 1981, 1983, 2000; Vigotsky, 1984).

En el juego de roles los componentes de la actividad se presentan juntos. La orientación (¿cómo jugar?), la ejecución (¿qué tan comprensible es jugar?, ¿qué tan fácil o difícil es?) y el control (si lo hace de manera correcta). El adulto muestra al niño en un primer momento de qué manera es posible realizar la acción lúdica. Él propone la secuencia
Tabla 1

Diferencias entre el juego temático de roles y el juego libre

\begin{tabular}{ll}
\hline \multicolumn{1}{c}{ Juego temático de roles sociales } & \multicolumn{1}{c}{ Juego libre } \\
\hline *Conforma la plataforma & \\
indispensable para la formación de la & \\
personalidad responsable del niño & \\
*Permite el desarrollo de aspectos & *No permite \\
indispensables para la preparación & el desarrollo \\
escolar del niño: actividades & de la actividad \\
simbólica, voluntaria, reflexiva, & organizada \\
comunicativa desplegada y la & *Es una actividad \\
imaginativa & que se da por \\
*Posibilita que el niño se dé cuenta & acciones al azar \\
de las relaciones que existen en la & *Impide que el \\
sociedad & niño seleccione \\
*Facilita que el niño identifique & estímulos \\
conscientemente las reglas, las & relevantes de los \\
obligaciones y los deberes que se & irrelevantes \\
deben cumplir & *No ayuda en el \\
*Permite que el niño regule su & reconocimiento \\
actividad y se someta a las reglas & de reglas de la \\
y a las situaciones de la sociedad & interacción social \\
*Posibilita la formación de los & $*$ No ayuda en \\
motivos e intereses & la formación \\
*Facilita centrar la atención y realizar & de motivos e \\
una tarea sin distracciones & intereses \\
*Posibilita la formación del objetivo & $*$ No permite que \\
de la actividad, tanto individual como & el niño adopte una \\
compartida & propia posición \\
*Permite el desarrollo de la compasión & moral \\
y el sentido personal & $*$ No permite \\
*Facilita que el niño desarrolle una & la solución de \\
propia posición moral para identificar & conflictos \\
comportamientos positivos y & \\
negativos & \\
\hline & \\
de conflicto & \\
&
\end{tabular}

de acciones lúdicas. Después el niño despliega la actividad con ayuda de su propio lenguaje verbal externo (Solovieva \& Quintanar, 2012).

\footnotetext{
El juego debe comprenderse como una actividad y no como un conjunto de movimientos y acciones libres y espontáneas del niño. Lo anterior implica que el jue-
}

10 Las neoformaciones son nuevas formaciones que aparecen en una edad que permiten el desarrollo de nuevas formaciones en la edad siguiente (Elkonin, 1980; Salmina \& Filimonova, 2001; Solovieva, Quintanar \& Lázaro, 2006) 
go posee la misma estructura que cualquier actividad humana: tiene motivo, base orientadora de la acción, conjunto de acciones y operaciones y medios (Talizina, 2000, p. 28).

Para Vigotsky "el juego temático de roles sociales es la fuente del desarrollo del niño y crea la zona de desarrollo próximo" (1984, p. 74). En este juego, el niño siempre se comporta arriba de su propia edad. Cuando el adulto le ayuda al niño a tomar los roles en el juego, se amplía su zona de desarrollo próximo. ${ }^{11}$ Es así como el juego de roles se constituye en una actividad transitoria que pasa de compartida dependiente hacia la realización independiente de acciones porque el niño juega de acuerdo con su propio deseo. Aquí, las acciones lúdicas se hacen más complejas.

Toda función psicológica en el desarrollo cultural del niño aparece en escena dos veces, en dos planos; primero en el plano social y después en el psicológico, al principio entre los hombres como categoría interpsíquica y luego en el interior del niño como categoría intra-psíquica. Las acciones internas se refieren a la posibilidad de hacer análisis mental (Vigotsky, 1995a, p. 150).

En el juego temático de roles, se pueden incluir diferentes relaciones sociales. Aquí, el niño retoma relaciones sociales de su cultura y los usos de los objetos que observa. Es por esto que se afirma que en el juego de roles el niño adquiere la dinámica de las relaciones sociales existentes por medio de su participación activa como personaje dentro de una temática establecida similar a la vida cotidiana.

El juego de roles influye en el ámbito de las actividades humanas y las relaciones entre las personas y su contenido fundamental es el hombre y las relaciones entre los adultos, en virtud de lo cual el juego es una forma de orientar las motivaciones de la actividad humana. Las relaciones sociales que los niños entablan en el juego han revelado sus funciones en el desarrollo psíquico de los preescolares (Elkonin, 1980, p. 20).

El juego temático de roles sociales es una actividad rectora en la infancia que permite asimilar la experiencia cultural para participar en la vida social. La actividad rectora es la que determina los cambios básicos en la psique del niño en una edad dada, en la cual surgen, se forman y reconstruyen los procesos psíquicos particulares o neoformaciones (Solovieva \& Quintanar, 2008). El juego de roles requiere de varios participantes, así como de la introducción, el desarrollo de un tema determinado y el despliegue de situaciones relacionadas entre sí, lo que prescribe el contenido concreto del juego.

Los niños cada vez incrementan el tiempo de juego, de esta manera se hace más profundo y duradero. De manera gradual surge la iniciativa de los niños a nivel verbal, poco a poco inician en el desarrollo de su propia voz narrativa para representar las situaciones. El adulto ayuda y participa, y en momentos críticos realiza acciones específicas si los niños no saben qué hacer, también puede modelar ciertos comportamientos. Es importante que el adulto identifique los momentos críticos para que inicie proponiendo una idea, o un personaje nuevo para que el juego reviva. Algunos elementos que son clave para el desarrollo del juego son el tema motivante, la participación activa en el rol, la inclusión emocional del adulto y los niños, la interacción en el diálogo, la tensión dramática del contenido, tener un argumento secuencial en forma de suspenso. El acontecimiento lúdico se construye en el diálogo. Los niños aceptan el juego solo si tiene sentido, solo si algo interesante ocurre (Bredikyte, 2004, p. 8).

El juego temático de roles sociales incluye elementos y procedimiento de realización así como se muestra en las figuras 1 y 2 . Es necesario considerar estos aspectos en el momento de iniciar el juego de roles con un grupo de niños. De esta manera, se podrá alcanzar éxito en la ejecución de esta importante actividad.

11 La utilización de la zona de desarrollo próximo requiere de la selección de acciones (tareas), las cuales son nuevas (desconocidas) y, al mismo tiempo, accesibles para el niño, es decir, se encuentran en su zona de desarrollo próximo. La zona de desarrollo próximo determina lo que el niño no puede realizar por sí solo y lo que puede realizar en la actividad conjunta y compartida con los adultos (Solovieva, 2004, p. 153). 


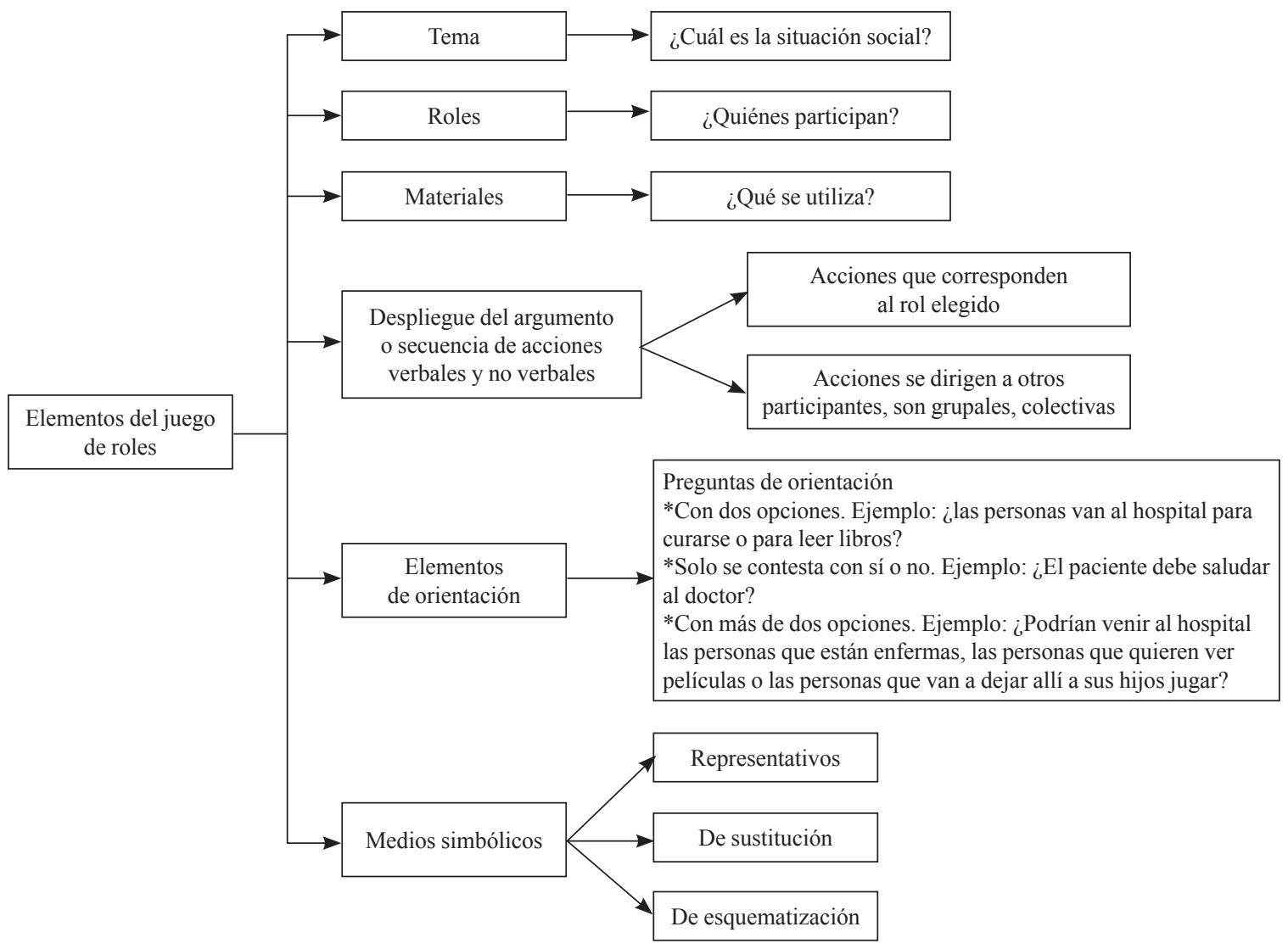

Figura 1. Elementos del juego de roles

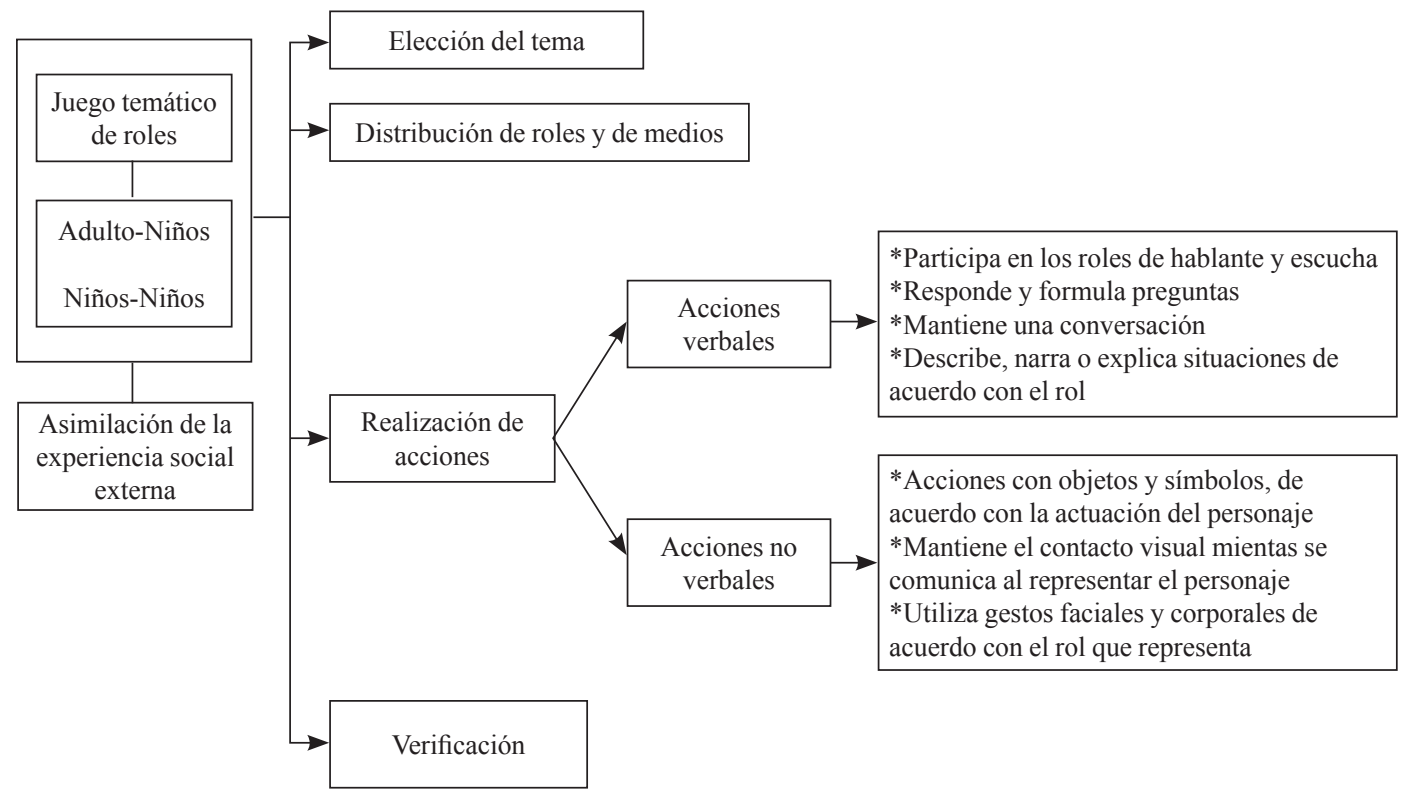

Figura 2. Procedimiento del juego de roles 


\section{El juego temático de roles sociales en el desarrollo de la actividad simbólica}

"La representación simbólica en el juego es, en esencia, una forma peculiar del lenguaje"

Vigotsky

La función simbólica es la característica esencial del proceso psíquico (Salmina, 1988; Solovieva \& Quintanar, 2012; Vigotsky,1995b). En educación inicial, la función simbólica se refiere a la posibilidad del niño de utilizar diversos medios (materializados, perceptivos y verbales) en su actividad predominante: juego. Aquí, el niño como ser social, asimila la experiencia que se ha acumulado en la cultura. El proceso de asimilación implica la realización de acciones cognoscitivas (Solovieva \& Quintanar, 2012; Talizina, 2009). "La primera representación simbólica se relaciona con el lenguaje con el cual se forman todos los restantes significados simbólicos de los signos" (Hetzer, citado por Vigotsky, 1995, p. 193).

Es necesario señalar que Vigotsky consideraba que en la edad infantil el juego es el camino más importante de todo el desarrollo cultural, en donde se subraya el desarrollo simbólico. El paradigma histórico-cultural gira alrededor del desarrollo simbólico, de la función psicológica de la cultura. La cultura acumula, crea, recrea, modifica y reproduce distintos tipos de símbolos y signos. Sin la presencia de la cultura, el desarrollo psicológico del niño no es posible (Solovieva \& Quintanar, 2012).

De acuerdo con Vigotsky (1995a, p. 18) la creación y el uso de símbolos y signos, constituye la particularidad y el medio principal del desarrollo cultural del hombre, y la base de la estructura de las formas culturales del comportamiento, es la actividad mediatizada. A este respecto, Vigotsky (1996a, p. 45) decía:

\footnotetext{
que durante el proceso de la vida social, el hombre creó y desarrolló los sistemas complejos de relaciones psicológicas, que de acuerdo con su naturaleza, son los signos, es decir, son estímulos creados artificialmente, cuyo papel es influir sobre el comportamiento y formar nuevas relaciones convencionales en el cerebro del hombre.
}

Vigotsky (citado por Zinchenko, 2005) distinguió entre un signo y una herramienta de la siguiente manera: una herramienta media las acciones de un individuo con objetos, o sea, se dirige hacia afuera, desempeña el papel de cambiar el objeto, sirve como medio para la actividad exterior del individuo que pretende dominar la naturaleza. Un signo no cambia en nada el objeto de la operación. En cambio, el signo representa un medio para influenciar el comportamiento de uno mismo o de otra persona. El signo se constituye en un medio de actividad interior dirigida a la autorregulación (Vigotsky, 1995a, 1996). Por tanto, el signo se dirige hacia adentro. Estas características no solo se relacionan con signos sino también con símbolos y palabras. La palabra dirigida a la resolución de un problema no solo se relaciona con los objetos presentes en el mundo exterior, sino también con el mismo comportamiento del niño, sus acciones e intervenciones (Zinchenko, 2005). "No hay individuos culturalmente autorregulados sin la estructura social emergiendo primero" (Vigotsky, 1995a, p. 36). Aquí, la consciencia se entiende por medio de la mediación semiótica dentro de un nivel metapsicológico (Robbins, 2005).

\footnotetext{
Una vez interiorizadas las funciones psicológicas superiores se convierten en sujeto del control voluntario. En palabras de Vigotsky (1984) "las acciones voluntarias empiezan solo cuando se perfecciona el comportamiento propio mediante un estímulo simbólico”. El más incuestionable de los sistemas simbólicos creados por la cultura humana (más no el único) es el lenguaje (Leontiev, 2000, p. 31).
}

Se plantea que puede existir el uso consciente y razonable de medios simbólicos al final de la edad preescolar, esta situación favorece a la conformación del plano ideal en la consciencia del niño. Sin el plano simbólico no puede conformarse el plano de representación ideal del mundo cultural en la consciencia del niño (Solovieva \& Quintanar, 2012)

El símbolo no representa el uso cultural del objeto mismo sino un contenido distinto que depende del objetivo. No existe nunca un contenido fijo. La formación del símbolo constituye el plano 
ideal de la acción, esta es una óptima posibilidad para preparación para la escuela como prevención de dificultades que pueden surgir en el aprendizaje escolar (Solovieva \& Quintanar, 2012, 2013).

De acuerdo con Elkonin, se proponen distintas acciones de juego de roles que implican la realización del rol a nivel objetal, representativo-simbólico y verbal. En este proceso siempre se incluye el inicio de la actividad voluntaria y la regulación verbal porque durante el juego siempre es permitido y prohibido hacer algo (Solovieva \& Quintanar, 2012). El uso de medios simbólicos puede ampliar la adquisición de conceptos empíricos (Salmina, 1988, 1989).

Es muy importante alcanzar la habilidad para utilizar los medios de los símbolos y signos en la actividad (Elkonin, 1989; Salmina, 1988). Para Salmina en la transición de la etapa preescolar a la escolar en el niño debe estar formado el tipo de actividad de símbolos y signos. Estos niveles son: (a) la sustitución, que representa el uso de sustitutos que realizan la misma función que el objeto que se reemplaza.; (b) la codificación y decodificación, que se refiere a la habilidad para expresar el fenómeno o acontecimiento de acuerdo con las reglas determinadas; (c) la esquematización, tiene que ver con la posibilidad de representar los objetos, los fenómenos y las situaciones con ayuda de modelos abstractos para clarificar las relaciones diversas posibles del problema a resolver representando un plan para su solución; (d) la generalización, que permite el uso del objeto, del gesto y la expresión en diversas situaciones; (e) la modelación, en los preescolares se presentan tres tipos de modelos: los que reflejan la estructura del objeto dado, los que reflejan la clase de objetos y los modelos simbólicos condicionales que reflejan las relaciones no concretas (Solovieva \& Quintanar, 2012; Talizina, 2000, 2009). (Ver figura 3)

El juego incluye etapas en su desarrollo (Solovieva \& Quintanar, 2012). Al inicio, se presentan acciones inespecíficas con los objetos, después el

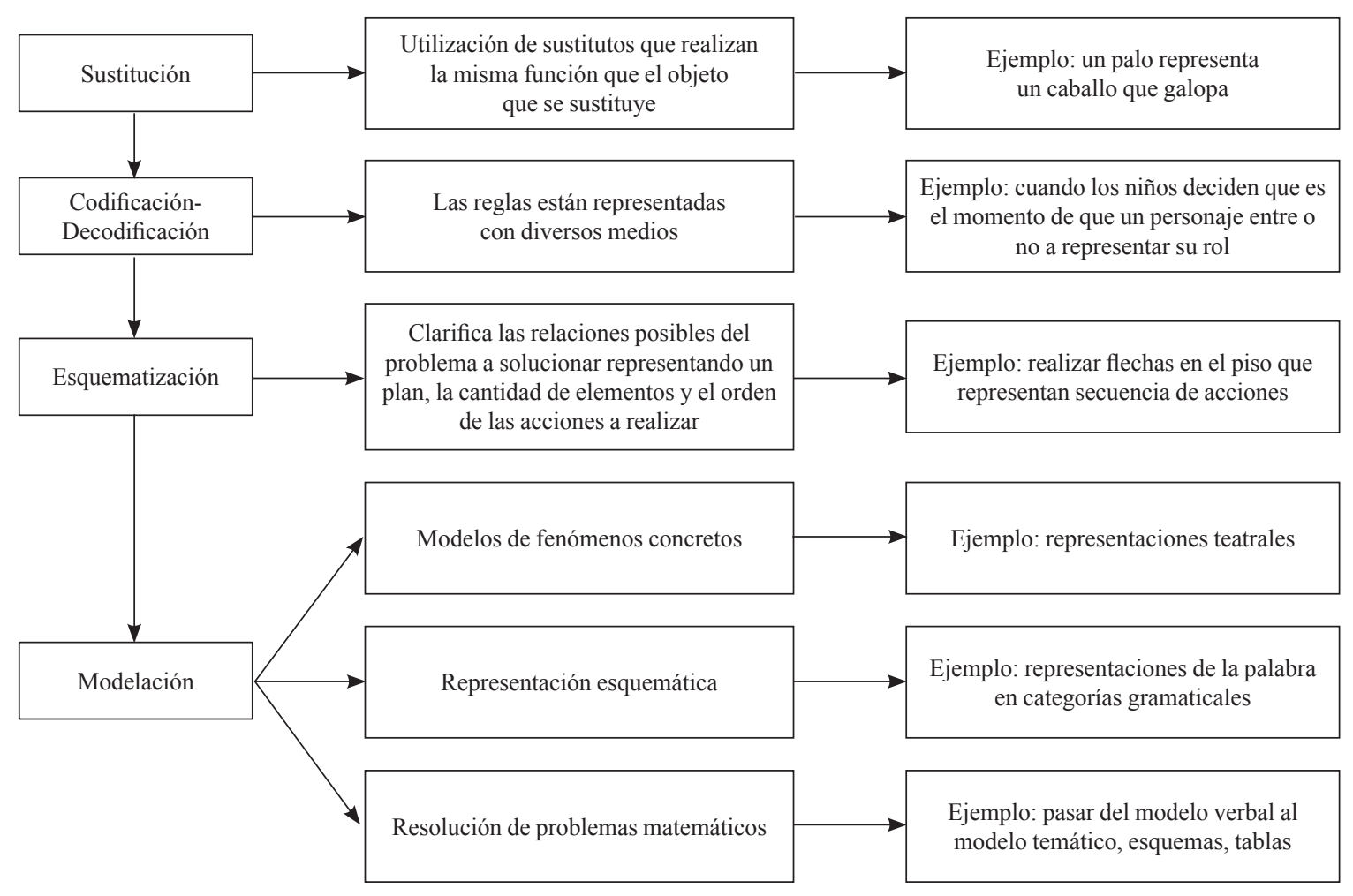

Figura 3. Niveles de la función simbólica en la transición de la edad preescolar a la escolar 
niño atribuye significado a sus acciones con los objetos con palabras, luego se forma la imagen interna por medio de la sustitución de objetos por otros y finalmente surge el motivo e interés por participar en las relaciones sociales. En este proceso se presentan actividades colaborativas en busca de un objetivo, en donde los objetos logran transformarse de manera infinita con la imaginación que los niños imprimen en sus juegos. Con el uso de objetos, la acción es muy similar a la situación real, luego las acciones y los roles adoptan una forma creativa que se despliega con el uso del lenguaje. De esta manera, el rol que asume el niño también se va transformando de forma amplia. Los niños que hacen parte del colectivo del juego de roles plantean y ponen en práctica ideas atractivas e interesantes. Al representar un rol pueden cambiar lo que les rodea de forma intencional y atribuyen significado a lo que dicen y hacen. Por medio de los roles, se pueden crear situaciones diversas. Por esta razón, se puede afirmar que en el juego de roles sin lugar a dudas la función simbólica se complejiza. En la figura 4 se presentan las etapas del desarrollo de la actividad de juego.

La identificación de características en los objetos en un primer momento permite la formación de conceptos. Estos son importantes porque permiten que el niño identifique de un grupo algunos elementos específicos. La identificación, modelación perceptiva y comparación con modelos son acciones perceptivas que permiten el desarrollo de la actividad de aprendizaje con facilidad (Solovieva \& Quintanar, 2012). El desarrollo de la percepción es importante para la formación del pensamiento en imágenes, para la formación de pensamiento lógico y del pensamiento científico.

Elkonin decía que los objetos pueden ser lo que se desee durante el juego. Los objetos pueden sustituir a otros de maneras muy distintas de acuerdo con las necesidades propias del juego. Un mismo objeto puede utilizarse de manera creativa de acuerdo con la iniciativa del niño. El niño puede

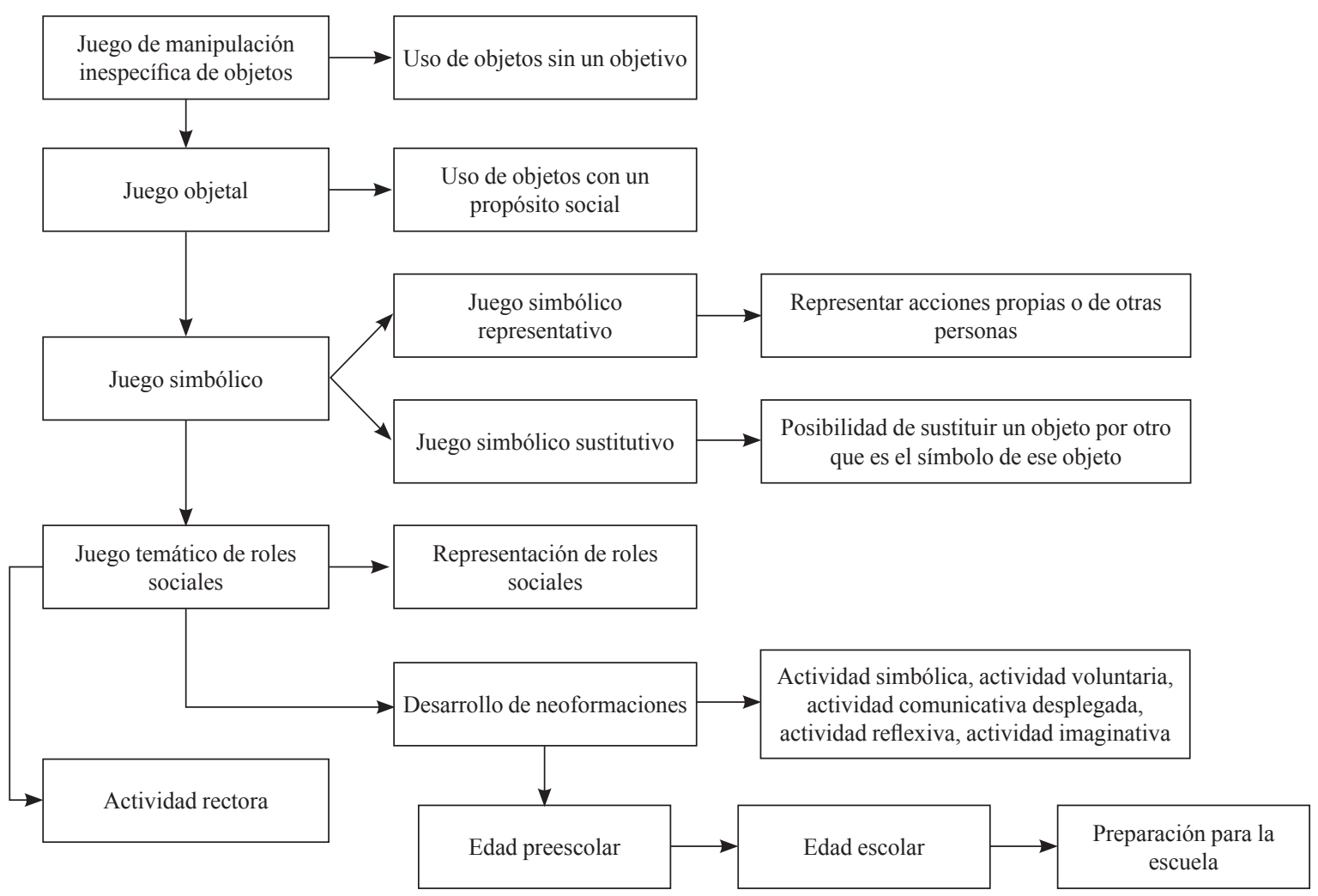

Figura 4. Etapas de desarrollo de la actividad de juego 
inventar distintas situaciones con el objeto. El niño crea situaciones indeterminadas con los objetos, los objetos se vuelven multifuncionales. Por ejemplo, un esfero podría ser una varita mágica si la usa el mago o una jeringa que usa una enfermera en el juego de roles del hospital.

El juego temático de roles sociales no puede surgir sin un desarrollo previo tanto del juego de manipulación de objetos, que constituye la generalización y la abstracción de las acciones objetales y su uso con sentido social, como del juego simbólico que implica la posibilidad de generalizar las acciones adquiridas dentro del juego objetal (Solovieva \& Quintanar, 2012). Las acciones objetales se perfeccionan, se generalizan y se abstraen de su contenido concreto y el niño las puede representar en cualquier contexto de manera simbólica y generalizada. Es así como predomina el carácter abstracto de la acción y su sentido sobre la base material (p. 41).

La estructura de los juegos cambia: va de los juegos compuestos por una serie de episodios, a menudo desligados, a convertirse en juegos con argumento que se va complejizando y desplegando de manera más metódica cada vez (Elkonin, 1980, p. 164).

En el proceso de interpretación de su papel, el niño transforma sus acciones y establece sus relaciones y la actitud ante la realidad (Elkonin, 1980). Es así como el niño crea situaciones imaginarias con sus coetáneos, ejecuta acciones y establece relaciones que forman parte de la vida cotidiana por medio del personaje que representa. En este proceso, los objetos transforman su significado porque al principio son usados como objetos de acuerdo con el uso cultural que se les da en la sociedad, pero después empiezan a transformarse en sustitutos de otros de forma variada y creativa. Asimismo, "el juego es un rol en desarrollo, las reglas son la escuela de la voluntad (el trabajo del escolar) y la situación imaginaria o ficticia es el camino de la abstracción" (Vigotsky, 1997, p. 88).

Los niveles de adquisición de la función simbólica se presentan en acciones materializadas, acciones perceptivas simbólicas y acciones verbales simbólicas. Las acciones materializadas hacen re- ferencia al uso del objeto de la acción como objetos concretos que sustituyen a los objetos; las acciones perceptivas simbólicas tienen que ver con la realización de acciones en el plano gráfico y esquemas $y$, las acciones verbales se refieren al aspecto lingüístico de la acción. La acción se transforma en conversación narrativa. En un primer momento, la acción verbal es reflejo de la acción realizada con el objeto a la cual el niño se refiere y representa. En este proceso se usan las palabras, los movimientos, los gestos. Después la acción verbal proyecta y ordena la conducta del niño, así como la de los demás en la interacción social.

En conclusión, se puede decir que inicialmente surge una acción simbólica aislada cuando el niño propone sustituir un objeto por otro, después el niño logra relacionar entre si dos acciones, después surgen cadenas de acciones simbólicas, a continuación surge el nivel simbólico desplegado promovido por iniciativa propia del niño. Este es el nivel óptimo para involucrar al niño en el juego de roles sociales, para incluirlo en interacción amplia con otros niños (Solovieva \& Quintanar, 2012).

\footnotetext{
La ejecución simultánea de acciones diversas con un mismo fin prepara la aparición de una imagen futura y generalizada de la situación. Las acciones objetales se perfeccionan, se generalizan y se abstraen (se separan) de su contenido concreto y el niño las puede representar en cualquier contexto de manera simbólica y generalizada. Así, empieza a predominar el carácter abstracto de la acción y su sentido, sobre su base material. Surge la utilización de signos externos e internos. De esta forma, el juego prepara el paso de los signos culturales externos al plano interno, en el cual el niño aprende a modificar y transformar, no solo las situaciones del mundo real, sino también su propia conducta y su mundo interno (Solovieva \& Quintanar, 2012, p. 27).
}

\section{El juego temático de roles sociales en el desarrollo de la actividad voluntaria}

La actividad voluntaria es considerada como la línea básica y central del desarrollo de la personalidad del niño (Salmina \& Filimonova, 2001; Solovieva \& Quintanar, 2010). La actividad voluntaria 
se refiere a la capacidad del niño para subordinar sus acciones a diversas instrucciones verbales del adulto y, paralelamente, desarrollar la capacidad para subordinar sus acciones a las acciones que ha formado en su propio lenguaje (Luria, 1979, 1982, 1989). Si la actividad voluntaria del niño no está organizada, no se fija su atención (Luria, 1986).

\begin{abstract}
El niño preescolar aprende gradualmente a someterse a sus acciones al control de su voluntad. En el momento en que alcanza aquel nivel de desarrollo, cuando en cierto grado puede someter sus acciones a un objetivo determinado, entonces se puede decir que dio un paso importante hacia la asimilación consciente de los conocimientos y las habilidades complejas en las condiciones de la enseñanza escolar (Neverovich, citado por Solovieva \& Quintanar, 2010, p. 189).
\end{abstract}

En el juego temático de roles, el niño puede desarrollar habilidades de planificación, organización y control de la actividad. En el juego aparece la orientación completa de la actividad y es comprendida por el propio niño, así como la regulación verbal de la acción, la habilidad para actuar de acuerdo con la temática, roles y reglas establecidas, la habilidad para inhibir acciones inadecuadas y la habilidad para mantenerse en el juego (Solovieva \& Quintanar, 2012). Es así como el niño logra verificar qué hacen los niños y si cumplen las reglas. Esta adquisición se da por medio de la interacción social, por medio de la observación de lo que hace el otro, primero en el plano externo y después en el interno. En este sentido, se puede afirmar que el juego temático de roles sociales posibilita el desarrollo de la consciencia humana (Vigotsky, 1995a; Luria, 1997).

En el juego el niño no solo actúa o desempeña algún rol, también se enfrenta a las consecuencias de sus acciones. Este hecho tiene particular interés debido a que la formación del plano interno del pensamiento nace a partir del sistema de relaciones humanas y no a partir del sistema de las relaciones materiales (Obukhova, citado por Bonilla, Solovieva, Figueroa, Martínez \& Quintanar, 2004, p. 120).
Para Vigotsky existen tres tipos de experiencias que conducen al desarrollo de la actividad voluntaria: ser regulados por otra persona, regular a otras personas y la auto-regulación. El juego temático de roles sociales mezcla el proceso que se da en estas experiencias (Solovieva \& Quintanar, 2012). La actividad voluntaria determina la estructura de la actividad de juego de roles, refleja cómo se lleva a cabo y sirve de mecanismo para su control.

\footnotetext{
Mediante la atención voluntaria se selecciona la información necesaria, se formula una estrategia de acción y se mantiene un control permanente sobre este. Todo esto se hace con la participación del lenguaje, el cual, por tanto, se incluye en la estructura psicológica de esta neoformación. La actividad voluntaria permite recodificar la información que llega, separa los eslabones más importantes de las acciones (crea un sistema interno de acción que se hace dominante), invalida las asociaciones colaterales de los estímulos directos del medio y de las huellas no útiles de la experiencia anterior (inhibe reacciones colaterales e inadecuadas), vigila la marcha de la acción (compara la acción realizada con el propósito inicial, si concuerda o no), controla su efectividad (corrige los errores, interrumpe la actividad cuando se ha cumplido el objetivo o la reanuda si no se ha logrado) (Álvarez, 2004, p. 58).

\section{El juego temático de roles sociales en el desarrollo de la actividad reflexiva}

Por medio del juego de roles el niño asimila la esencia de las relaciones sociales y se contribuye con su desarrollo emocional, lo que tiene impacto en su personalidad reflexiva. Esta actividad organiza la actividad cognitiva del niño. Siempre son juegos creativos que se garantizan con una serie de condiciones. Para su desarrollo, se considera el interés del niño y su edad psicológica.

La actividad reflexiva se define como la capacidad de reformular el propio pensamiento, mediante el uso del lenguaje, lo que lleva a reflexionar sobre el comportamiento de sí mismo y las acciones de los demás, facilitando la transformación del sentido mismo del intercambio social, habilidad compleja de la mente que impone demandas simultáneas sobre el individuo respecto a sus expresiones verbales 
y sus acciones voluntarias, al hacerse consciente de ellas (González-Moreno, 2009).

La personalidad reflexiva es la característica de la psique que se manifiesta en todos los tipos de actividad (Talizina, 2009). Esta característica posibilita que el niño se incluya en las auténticas relaciones sociales de manera positiva y en la edad escolar ayuda a la formación de hábitos responsables frente a la realización de las diferentes actividades.

En el diálogo que se establece en la conversación entre los participantes del juego de roles, el niño clarifica y re-elabora sus ideas (representaciones mentales) usando el lenguaje. Ese proceso se internaliza y permite que se usen de forma reflexiva los medios simbólicos tanto externos como internos. Además, se genera nuevo contenido de ideas de acuerdo con las temáticas y a los roles que se representan. Los niños deben pensar qué decir y cómo expresase. Esto tiene que ver con la manera de codificación o representación de ideas y el subsecuente uso del lenguaje narrativo en situaciones aplicables.

En la actividad del juego de roles se producen emociones positivas que facilitan en el niño la adopción de un comportamiento moral y una conducta responsable ante los demás, ante sí mismo y ante la actividad que se realiza.

La actividad reflexiva se refiere a anticipar el comportamiento de sí mismo. De acuerdo con Bajtin y Vigotsky solo por medio del otro que es un espejo para el niño, él se empieza a conocer a sí mismo.

\section{El juego temático de roles sociales en el desarrollo de la actividad comunicativa desplegada}

El juego temático de roles sociales es la actividad que permite que el niño (inicialmente de manera emocional y después de manera intelectual) adquiera el sistema de comunicación, además de ser la forma específica de adquisición de la realidad (Bonilla, Solovieva, Figueroa, Martínez \& Quintanar, 2004).

Indudablemente la comunicación es un indicador de preparación del niño para la escuela. Durante la comu- nicación se forma la habilidad del niño para someterse a las reglas y orientarse hacia las normas sociales. Asimismo, esta habilidad permite la comprensión recíproca a nivel social (Talizina, 2009, p. 48).

La actividad lúdica es la fuente para el desarrollo y la habilidad para comunicarse, sobre la base de la interacción social. Si el niño interactúa bien en la actividad de juego entonces se podría predecir una interacción adecuada en la actividad de aprendizaje escolar (Alkutina, 2002). Esto permite la formación de conceptos de acuerdo con Vigotsky. Si el niño no sabe interactuar en el juego entonces se podrían presentar problemas en la edad escolar.

Los niños se diferencian de acuerdo con el éxito en el aprendizaje que depende de la habilidad para interactuar con otros niños y con el pedagogo (Alkutina, 2002). Esto se manifiesta en la iniciativa del niño para conversar, para comunicarse. Por esta razón, se afirma que la actividad comunicativa es muy importante para el desarrollo de la iniciativa. Si el niño no tiene iniciativa esto dificulta toda su comunicación. En el juego se forma una posibilidad de interacción comunicativa. Esto garantiza el desarrollo de la creatividad, que tenga entusiasmo y sea apoyado. Además, el juego de roles permite que se genere la iniciativa en el niño para resolver problemas. También se generan y resuelven conflictos en relación con los roles que asumen. Esto posibilita que los niños en la escuela primaria resuelvan diferentes tipos de problemas con métodos distintos.

Desde la teoría de la formación de acciones por etapas (Galperin, 1976), la formación de la actividad comunicativa se inicia desde la actividad material con los objetos. Esta habilidad poco a poco se va complejizando, lo que se evidencia en el juego temático de roles cuando se trabaja adecuadamente.

Uno de los indicadores de la preparación para la escuela es el nivel de comunicación de los niños preescolares (Kravtsova, 1981, Lisina, 1986). Debido a que el sometimiento a las reglas y la orientación hacia las normas sociales se forma en la comunicación, entonces el indicador de la formación de preparación puede ser el nivel de formación de la comunicación. La ausencia de preparación, en este caso, se descubre en el hecho de 
que el niño no puede realizar las exigencias, incluirse en la actividad de aprendizaje, en la comunicación con coetáneos, con el maestro, realizar todas las instrucciones (Salmina, 2010, p. 71).

Por medio de la actividad verbal que se presenta en el juego temático de roles sociales, surge la conexión entre un ser humano y otro. Aquí, el lenguaje adquiere sentido social y coherencia, dado que surge la motivación para la comunicación y la posibilidad para regular su propia actividad por medio del mismo lenguaje (Solovieva \& Quintanar, 2012). Este proceso permite el intercambio de información y la negociación de significados al comunicar o compartir significados. Asimismo, posibilita participar en los roles de hablante y oyente, al desarrollar la habilidad para tomar la palabra asumiendo turnos conversacionales y relacionar de forma coherente los turnos, todo esto en el marco del uso de mecanismos de cohesión y de mantenimiento del tópico.

El adulto cumple un rol muy importante porque en un primer momento es quien organiza y dirige la actividad de juego temático de roles sociales. Por esta razón, es necesario relacionar las producciones entre sí, es decir, se debe lograr la pertinencia interaccional para que el niño tenga mayores posibilidades de desplegar sus acciones, representaciones y expresiones lingüísticas. También es necesario ayudar al niño a negociar significados entre lo que se hace y dice durante el juego. Es así como se hace cada vez más fácil y divertido participar en la actividad lúdica a nivel social.

Los componentes estructurales de la actividad comunicativa en el juego temático de roles sociales son los que se presentan en la figura 5, los cuales están basados en Salmina (2010).

En el proceso de la comunicación durante la interacción social, sobre la base de las relaciones humanas en el juego de roles, surge la función simbólica. Esto se caracteriza por el hecho de que esta función primero es social y después es interna. Como resultado, la actividad simbólica se hace más compleja. Como lo proponen Solovieva y Quintanar (2010) "al retomar uno u otro papel del juego, el niño retoma también aquellas relaciones internas de la conducta que se incluyen en el papel dado y en otros tipos de su actividad" (p. 21).

1. El objeto de la comunicación son los coetáneos y el adulto.

2. La necesidad de comunicarse consiste en la posibilidad de que los niños sean valorados asumiendo sus roles por los demás participantes del juego.

3. Los motivos de la comunicación se refieren a aquello por lo cual se establecen diálogos entre los participantes del juego por medio de los roles que se interpretan y las temáticas consideradas.

4. La acción de comunicación se dirige a los demás. Aquí se tienen en cuenta las acciones de iniciativa y las acciones de respuesta al participar en los roles de hablante y escucha.

5. Los problemas de la comunicación que se pueden generar en el juego de roles se deben a fallas en la negociación de significados que tienen los participantes y a fallas en el campo de atención intersubjetiva, es decir en aquello que alguno de los integrantes del juego supone que conoce otro participante.

6. Los medios de comunicación son las operaciones con cuya ayuda se realizan las acciones de comunicación. Esto se refiere específicamente a la conversación que se genera en el juego.

Figura 5. Componentes estructurales de la actividad comunicativa en el juego temático de roles sociales

\section{El juego temático de roles sociales en el desarrollo de la imaginación}

El niño en su participación activa en el juego de roles puede imaginar las características del personaje que representa para asumir activamente ese papel a nivel social en la interacción comunicativa. En un primer momento, requiere del apoyo del adulto y después logra desplegar su representación al inventar características sofisticadas y creativas.

En el juego de roles, los niños representan diversas situaciones imaginarias que hacen parte de la vida real y de la fantasía. La imaginación tiene un gran significado no solo para la actividad escolar, sino también para la educación de la personalidad creativa (Talizina, 2009).

La imaginación se encuentra en relación directa con la riqueza y variedad de la experiencia acumulada por el hombre (Vigotsky, 2007). Esto equivale 
a decir que cuanto más rica es la experiencia humana, tanto mayor será el material del que dispone esa imaginación. Para Vigotsky todas las formas de representación en la imaginación encierran en sí elementos afectivos. En otras palabras, todo lo que edifique la imaginación influye recíprocamente en los sentimientos.

Algunos de los indicadores que muestran un desarrollo adecuado de la imaginación en la edad preescolar son: (a) posibilidad para imaginar el resultado de la acción; (b) posibilidad para proponer de forma novedosa el uso de signos y símbolos en la propia actividad y (c) posibilidad para imaginar el resultado de la situación (Solovieva \& Quintanar, 2012).

\section{El sentido humano del juego temático de roles sociales}

El juego temático de roles sociales es un producto de la actividad en la cual el hombre transforma la realidad y modifica el mundo. Asimismo, el juego permite que el niño tenga la posibilidad de participar de forma genuina en el contexto social y cultural al hacer uso de recursos simbólicos.

El carácter del juego humano estriba en la aptitud de transformar la realidad. En el juego se forma y manifiesta la necesidad del niño de actuar sobre el mundo, o de influir en el. En ello estriba su fundamental y más general significación (Leontiev, 1983, p. 645).

La concepción del juego de roles en educación inicial se basa en la concepción de lo humano. El ser humano desde las etapas iniciales de vida se va configurando como un proyecto, y está abierto a la multiplicidad de formas sociales y culturales, lo cual lleva consigo una intrínseca intencionalidad: la realización de la persona humana en todas sus esferas del desarrollo: emocional-afectiva, comunicativa, comportamental y social (González-Moreno, Solovieva \& Quintanar, 2012).

En el juego de roles es importante considerar el tema y el contenido. El tema refleja las condiciones concretas de la vida del niño. El tema tiene un fondo social. El contenido del rol asumido por el niño es precisamen- te la reconstrucción de un aspecto de la realidad. El contenido del juego revela la penetración más o menos profunda del niño en la actividad de los adultos; puede revelar solo el aspecto externo de la actividad humana, solo el objeto con el que opera el hombre o la actitud que adopta frente a su actividad y la de otras personas o, por último, el sentido social del trabajo humano (Elkonin, 1980, p. 38).

El juego de roles es la actividad por la cual se extrae la esencia social propiamente humana, sus objetivos y normas de relación entre las personas (Elkonin, 1980). El juego de roles proporciona una atmósfera en donde los niños pueden aprender a resolver problemas. Posteriormente, cuando los niños se enfrentan a problemas más complejos en el mundo real, el aprendizaje que adquirieron durante el juego les proporciona grandes beneficios (Bruner, 1991). El juego es una proyección del mundo interior, en el que se interioriza el mundo externo hasta llegar a hacerlo parte de uno mismo (Bruner, 1984).

El juego se convierte en escuela para la vida. Naturalmente, el niño no juega a fin de prepararse para la vida, sino que en el juego adquiere por sí mismo esa preparación, porque regularmente siente la necesidad de ejecutar jugando precisamente aquellos actos que, aunque nuevos, todavía no se han convertido en costumbre. En el juego se desarrolla y se prepara para su ulterior actividad. El niño juega porque se desarrolla y se desarrolla porque juega. El juego es la práctica de su desarrollo (Rubinstein, 1967, p. 651).

\section{Reflexión final}

El juego temático de roles sociales asegura un óptimo desarrollo en la primera infancia. En el juego temático de roles sociales los niños desarrollan habilidades para interactuar con los demás, resolver problemas, identificar sus intereses, usar el lenguaje, retener y recordar la información. Sin embargo, en el preescolar es más frecuente la preocupación por las habilidades académicas en los niños que por su desarrollo lúdico. Como consecuencia, los niños no alcanzan cierto nivel de desarrollo psicológico de las neoformaciones que posibilitan y facilitan la transición a la edad escolar. Además, en la gran 
mayoría de las aulas de hoy no se utiliza este tipo de juego, lo que conduce a grandes dificultades en el desarrollo del niño.

Por esta razón surge la preocupación por apoyar a los educadores en pedagogía infantil con estrategias de cómo jugar con los niños. De esta manera, los preescolares alcanzarán el desarrollo adecuado por medio de su participación activa en juegos de roles complejos. En este sentido, se hace necesario generar planeaciones pedagógicas innovativas que incluyan al juego de roles para lograr transformaciones dentro del aula de clase. Así se puede contribuir con la calidad educativa relevante y pertinente.

El pedagogo debe preguntarse si el niño jugó o no jugó en el nivel preescolar. Esto determina en gran medida si el niño está preparado o no para iniciar la etapa escolar. Aquí, se pueden observar indicadores positivos y negativos, si la edad transcurre de manera positiva o si hay desviaciones (Solovieva \& Quintanar, 2012).

Pensar en el juego de roles como actividad fundamental en la edad preescolar implica preocuparse por las aportaciones que se pueden realizar desde el quehacer pedagógico en el aula de clase para garantizar la formación de habilidades mínimas necesarias para el aprendizaje escolar y proporcionar las bases positivas de personalidad del niño y, en general, para la adquisición del sistema de conocimientos aplicables a la vida cotidiana. Para ello, se requiere de un trabajo intencional, sistemático, consciente y reflexivo a nivel pedagógico. En palabras de López-Calva (2003, p. 15):

\footnotetext{
el hecho educativo pretende de manera intencional que el ser humano desarrolle todas y cada una de sus potencialidades para buscar la construcción de un mundo mejor, porque siendo más humano se es más dueño de la propia actividad consciente, se es más libre y se toman mejores decisiones. Así se incrementan las posibilidades de participación activa y creativa en el contexto social y cultural.
}

Se trata de un juego de roles dirigido que posibilita el desarrollo de los aspectos característicos de esta etapa del desarrollo como la función simbólica, la imaginación, el sentido personal, el respeto por el otro, la actividad reflexiva, la actividad voluntaria. Aspectos que permiten que el niño sea más sensible para lograr que sea mejor persona y desarrolle su propia posición moral frente a situaciones diversas. El juego de roles también ayuda a que el niño entienda que los otros piensan diferente. En este sentido, se podría decir que el juego ayuda al aprendizaje de actitudes como la solidaridad y el respeto por la vida; posibilita la negociación en situaciones de conflicto.

Si en la edad preescolar no se desarrollan las neoformaciones: simbólica, voluntaria, reflexiva, comunicativa desplegada y la imaginación, se podrían predecir dificultades en la edad escolar para recordar términos, recuperar información, representar datos matemáticos, resolver problemas, comprender textos literarios, hacer inferencias, análisis e interpretaciones de textos, producir textos argumentativos y expositivos. Esto se presenta porque el niño no tiene la posibilidad de hacer representaciones simbólicas en el nivel complejo, no sigue instrucciones, no logra mantener el objetivo de la actividad, no logra reflexionar acerca de sus propias acciones. Como consecuencia, en el niño se genera un efecto negativo a nivel emocionalafectivo que muchas veces se refleja en comportamientos inadecuados.

Se concibe que las diferentes formas de juego pueden y deben aplicarse en la institución escolar. De acuerdo con el planteamiento de Solovieva y Quintanar (2012) "al final de la edad preescolar cada niño debe tener su experiencia propia de las acciones con signos y símbolos (acciones simbólicas) y en el juego se puede brindar esta opción" (p. 120). Es así como se evita que el niño presente dificultades para comprender textos y su nivel sea bajo en la adquisición conceptual en las diferentes materias que hacen parte de los programas curriculares.

Como indica Elkonin (1980) "al conocer con profundidad el juego temático de roles sociales y su influencia en el desarrollo infantil, se puede facilitar la dirección pedagógica y la formación de esta importantísima actividad en el niño" (p. 163). Esa es la apuesta que conduce a mejorar las condiciones de vida de los niños al reconocer las realidades sociales de la población.

En conclusión, se considera que el juego temático de roles sociales posibilita el logro de la calidad 
de la educación desde el preescolar, entendiendo el planteamiento del Ministerio de Educación Nacional (2013, p. 1):

La calidad en la educación es aquella que forma mejores seres humanos, ciudadanos con valores éticos, respetuosos, que ejercen los derechos humanos, cumplen con sus deberes y conviven en paz. Una educación que genera oportunidades legítimas de progreso y prosperidad. Una educación que es pertinente, que contribuye a cerrar brechas de inequidad y en la que participa toda la sociedad (2013, p. 1).

\section{Referencias}

Akhutina, T. \& Pilayeva, M. (2012). Overcoming learning disabilities: A Vigotskian-Lurian neuropsychological approach. Cambridge: Cambridge University Press.

Akhutina, T. V. (2002). Diagnóstico y corrección de la escritura. Revista Española de Neuropsicología, 4 , 2-3.

Álvarez, M. (2004). Neuropsicología de la alteración de la atención voluntaria. En Y. Solovieva \& L. Quintanar. Métodos de intervención neuropsicológica infantil (pp. 47-90). Puebla: Benemérita Universidad Autónoma de Puebla. Colección Neuropsicología y Rehabilitación.

Bergen, D. (2002). The Role of Pretend Play in Children's Cognitive Development. Early Childhood Research \& Practice: An Internet Journal on the Development, Care, and Education of Young Children 4(1). Recuperado de http://ecrp.uiuc.edu/ v4n1/index.html

Bodrova, E. (1998). Development of dramatic play in young children and its effects on self-regulation: the Vigotskyan approach. Journal of early childhood teacher education, 19(2), 38-46.

Bodrova, E. \& Leong, D. J. (1996). Tools of the mind: The Vigotskyan approach to early childhood education. Englewood Cliffs, N. J., Merrill: Prentice Hall.

Bodrova, E. \& Leong, D. J. (2001). Tools of the mind: a case study of implementing the Vigotskyan approach in american early childhood and primary classrooms. Switzerland: International Bureau of Education.
Bodrova E. \& Leong, D. J. (2003). Learning and development of preschool children from the Vigotskyan perspective. En V. Ageyev, B. Gindis, A. Kozulin, S. Miller (Eds.). Vigotsky's theory of education in cultural context (pp. 156-176). New York: Cambridge University Press.

Bodrova, E. \& Leong, D. J. (2010). Currículum and play in early child development. En Tremblay RE, Boivin M., Peters RDeV, (eds.), Encyclopedia on Early Childhood Development (pp. 1-6) Montreal, Quebec: Centre of Excellence for Early Childhood Development and Strategic Knowledge Cluster on Early Child Development.

Bonilla, M. (2009). Los problemas de lenguaje, atención y memoria y sus repercusiones en la lectoescritura. En Quintanar, L., Solovieva, Y., Lázaro, E., Bonilla, R., Mejía, L., Eslava, J. et al. Dificultades en el proceso lectoescritor (pp. 51-69). México: Trillas.

Bonilla, M. (2013). Formación de la función simbólica en preescolares a través de las actividades de juego. (Tesis doctoral, Universidad Iberoamericana de Puebla, México).

Bonilla, M., Solovieva, Y. \& Jiménez, N. (2012). Valoración del nivel de desarrollo simbólico en la edad preescolar. Revista CES Psicología, 5(2), 56-69.

Bonilla, R., Solovieva, Y., Figueroa, S., Martínez, J. \& Quintanar, L. (2004). Tratamiento neuropsicológico en niños con TDA con predominio de impulsividad. En Y. Solovieva, \& L. Quintanar. Métodos de intervención neuropsicológica infantil (pp. 117-146). Puebla: Benemérita Universidad Autónoma de Puebla. Colección Neuropsicología y Rehabilitación.

Bredikyte, M. (2004). Role of Play in Child's Life [In Lithuanian]. Zvirbliu takas 4, 6-11.

Bredikyte, M. \& Hakkarainen, P. (2007). Cultural development of the child through narrative learning. Recuperado de http://www.centrepompi-dou.fr/ streaming/symposium/en/session3.htmBruner, J. (1984). Acción, pensamiento y lenguaje. Madrid: Alianza.

Bruner, J. (1991). Actos de significado. Más allá de la revolución cognitiva. Madrid: Alianza.

Cole, M., Hakkarainen, P. \& Bredikyte, M. (2010). Culture and early childhood learning. En Tremblay R. E., Barr R. G., Peters RDeV, Boivin M., (eds.) Encyclopedia on Early Childhood Development. 
Montreal, Quebec: Centre of Excellence for Early Childhood Development, 1-6. Recuperado de http://www.child-encyclopedia.com/documents/ Cole-Hakkarainen-BredikyteANGxp.pdf.

Consejo Nacional de Política Económica Social, República de Colombia, Departamento Nacional de Planeación, Política Pública Nacional de Primera Infancia. Colombia por la primera infancia. Documento Conpes social. Ministerio de la Protección Social, Ministerio de Educación Nacional, Instituto Colombiano de Bienestar Familiar, Bogotá. Recuperado de http://cms-static.colombiaaprende. edu.co/cache/binaries/articles-177828_archivo_ pdf_conpes 109.pdf?binary_rand $=3435$

Colombia aprende. La red del conocimiento. Preescolar, básica y media. (2011). Sistema educativo en Colombia. Bogotá: Ministerio de Educación Nacional. Recuperado de http://www.colombiaaprende. edu.co/html/home/1592/article-235863.html

Elkonin, D. B. (1995). Desarrollo psicológico en las edades infantiles. Moscú: Academia de Ciencias Pedagógicas y Sociales.

Davidov V. V. (1996). La teoría de la enseñanza que conduce al desarrollo. Moscú: Inter.

Elkonin, D. B. (1980). Psicología del juego. Madrid: Pablo del Río.

Elkonin, D. B. (1989). Obras escogidas. Moscú: Universidad Estatal de Moscú.

Galperin, P. Ya. (1976). Introducción en la psicología general. Moscú: Universidad Estatal de Moscú.

González-Moreno, C. X. (2009). La utilización de la actividad de juego temático de roles sociales en la formación del pensamiento reflexivo en preescolares. (Tesis de maestría, Pontificia Universidad Javeriana, Bogotá).

González-Moreno, C. X., Solovieva, Y. \& QuintanarRojas, L. (2009). La actividad de juego temático de roles en la formación del pensamiento reflexivo en preescolares. Magis, Revista Internacional de Investigación en Educación 2(3), 173-190.

González-Moreno, C. X., Solovieva, Y. \& QuintanarRojas, L. (2011). Actividad reflexiva en preescolares. Perspectivas psicológicas y educativas. Universitas Psychologica, 10(2), 423-440.
González-Moreno, C. X., Solovieva, Y., \& QuintanarRojas, L. (2012). Neuropsicología y Psicología histórico-cultural: aportes en el ámbito educativo. RevFacMed. 60(3), 177-187.

Hetzer, H. (1926). Die Siymbolische Darstellung in der frühen Kindheit. New York: Wien-Leipzig.

Lázaro, E. (2009). Identificación temprana de las dificultades para la actividad escolar. En L. Quintanar, Y. Solovieva, E. Lázaro, R. Bonilla, L. Mejía, J. Eslava et al. Dificultades en el proceso lectoescritor (pp. 23-34). México: Trillas. 23-34.

Lázaro, E., Solovieva, Y., Cisneros, N. \& Quintanar, L. (2009). Actividades de juego y cuento para el desarrollo psicológico del niño preescolar. Revista internacional Magisterio, 37, 80-85.

Leontiev, A. N. (1981). Problemas del desarrollo de la psique. Moscú: Universidad Estatal de Moscú.

Leontiev, A. N. (1983). Obras escogidas. Tomos 1 y 2. Moscú: Pedagogía.

Leontiev, A. N. (2000). Conferencias sobre psicología general. Moscú: Sentido.

Lineamiento Pedagógico y Curricular para la Educación Inicial en el Distrito. (2010). Secretaría Distrital de Integración Social y la Secretaría de Educación del Distrito. Bogotá: Secretaría Distrital de Integración Social y Alcaldía Mayor de Bogotá.

Lindqvist, G. (1995). The Aesthetics of Play: A Didactic Study of Play and Culture in Preschools. Stockholm: Almqvist \& Wiksell.

López-Calva, M. (2003). Pensamiento crítico y creatividad en el aula. México: Trillas.

Luria, A.R. (1979). Lenguaje y conciencia. Moscú: Universidad Estatal de Moscú.

Luria, A.R. (1982). El papel del lenguaje en el desarrollo de la conducta. México: Cartago.

Luria, A.R. (1986). Las funciones corticales superiores en el hombre. México: Fontamara.

Luria, A. R. (1989). El cerebro en acción. México: Ediciones Roca.

Luria, A. R. (1997). Conciencia y lenguaje. México: Visor.

Mikhailenko, N. \& Korotkova, N. (2001). Kak igrat s det'mi [How to play with children]. Moskva: Akademicheskij Project. 
Ministerio de Educación Nacional. (2013). Mejorar la Calidad de la Educación en todos los niveles. Ciudad Bogotá: Editorial Plan de Acción MEN 20132014. Recuperado de http://www.mineducacion. gov.co/1621/article-278740.html

Neverovich, Ya. Z. (1977). The Development of Motor Acts with Objects in the Preschool Child. Journal of Russian and East European Psychology. 16(1) 35-45.

Obukhova L. F. (1995). Psicología infantil. Teorías, hechos, problemas. Moscú: Trivola.

Obukhova, L. F. (2006). Psicología del desarrollo. Moscú: Educación superior.

Quintanar, L. \& Solovieva, Y. (2005). Análisis neuropsicológico de los problemas en el aprendizaje escolar. Revista Internacional del Magisterio 15 , 26-30.

Quintanar, L. \& Solovieva, Y. (2007). Neuropsicología y aprendizaje escolar. Revista Ciencia y Desarrollo $33,60-65$.

Robbins, D. (2005). Entendiendo la metapsicología de Vigotsky. Eclecta. Revista de Psicología General $3(9$ y 10$)$ 16-28.

Rubinstein, S. L. (1967). Principios de psicología general. México: Editorial Grijalbo.

Salmina, N. (1988). Signo y símbolo en la educación. Moscú: Universidad Estatal de Moscú.

Salmina, N. (1989). Tipos y formas de materialización de la enseñanza. Moscú: Universidad Estatal de Moscú.

Salmina, N. (2010). Indicadores de preparación de los niños para la escuela. En Y. Solovieva \& L. Quintanar. Antología del desarrollo psicológico del niño en la edad preescolar (67-74). Trillas: México.

Salmina, N. \& Filimonova, O. (2001). Diagnóstico y corrección de la voluntariedad en la edad preescolar escolar menor. México: Universidad Autónoma de Tlaxcala.

Schmelkes, S. (1994). Hacia una Mejor Calidad de Nuestras Escuelas. México: SEP. Biblioteca para la actualización del maestro.

Schmelkes, S. (1995). Hacia una Mejor Calidad de Nuestras Escuelas. INTERAMER 32 Serie educativa. Colección INTERAMERIINTERAMER Collection Recuperado de http://www.ctascon.com/ Hacia\%20una\%20mejor\%20calidad\%20de\%20 las\%20Escuelas.pdf
Schmelkes, S. (1997). Educación para la vida: algunas reflexiones en torno al concepto de relevancia de la educación. En Artículos sobre la educación básica, Documento DIE, 50, (pp. 5-13). México: DIE-CINVESTAV-IPN.

Solovieva, Y., Quintanar, L. \& Lázaro, E. (2006). Efectos socioculturales sobre el desarrollo psicológico y neuropsicológico en niños preescolares. Cuadernos Hispanoamericanos de Psicología 6(1), 9-20.

Solovieva, Y. \& Quintanar, L. (2007). Análisis neuropsicológico de la acción escolar desde el paradigma histórico-cultural. Revista de Psicología General y Aplicada 60(3), 217-234.

Solovieva, Y. \& Quintanar, L. (2008). Enseñanza de la lectura. Método práctico para la formación lectora. México: Trillas.

Solovieva, Y., Machinskaya, R., Quintanar, L., Bonilla, R. \& Pelayo, H. (2009). Neuropsicología del TDA en la edad preescolar. Puebla: Benemérita Universidad Autónoma de Puebla, Colección Neuropsicología y Rehabilitación.

Solovieva, Y. \& Quintanar, L. (2010). Antología del desarrollo psicológico del niño en edad preescolar. México: Trillas.

Solovieva, Y. \& Quintanar, L. (2012). La actividad de juego en la edad preescolar. México: Trillas.

Talizina, N. (2000). Manual de psicología pedagógica. México: Facultad de Psicología. Universidad Autónoma de San Luis Potosí.

Talizina, N. (2009). La teoría de la actividad aplicada a la enseñanza. Puebla: Benemérita Universidad Autónoma de Puebla, Colección Neuropsicología, Educación y desarrollo.

Vigotsky, L. S. (1984). El instrumento y el signo en el desarrollo del niño. Obras escogidas. Tomo 6. Moscú: Pedagogía.

Vigotsky, L. S. (1995a). Obras escogidas. Tomo III. Madrid: Visor.

Vigotsky, L. S. (1995b). El desarrollo del sistema nervioso. En L. Quintanar, (ed.). La formación de las funciones psicológicas durante el desarrollo del niño (pp. 161-178). México: Universidad Autónoma de Tlaxcala.

Vigotsky, L. S. (1996a). El desarrollo de los procesos psicológicos superiores. Barcelona: Crítica. Grijalbo Mondadori. 
Vigotsky, L. S. (1996b). Obras escogidas. Tomo 4. Madrid: Visor.

Vigotsky, L. S. (1997). Obras psicológicas escogidas. 5 vols. ( $2^{\mathrm{a}}$ ed.). Madrid: Aprendizaje-Visor.

Vigotsky, L. S. (2007). La imaginación y el arte en la infancia. Madrid: Akal.

Zinchenko, V.P. (2005). Desde la psicología clásica a la orgánica: En conmemoración del centenario del nacimiento de Lev Vigotsky. Eclecta. Revista de psicología general Vol III(9 y 10), 40-56. 